\title{
Subtypes and Symptomatology of Irritable Bowel Syndrome in Children and Adolescents: A School-based Survey Using Rome III Criteria
}

\author{
Shaman Rajindrajith* and Niranga M Devanarayana \\ Faculty of Medicine, University of Kelaniya, Ragama, Sti Lanka
}

\section{Background/Aims}

This study was conducted with objectives of assessing subtypes of irritable bowel syndrome (IBS) in children aged 10-16 years, their symptomatology and gender differences.

\section{Methods}

For this survey, 107 children who fulfilled Rome III criteria for IBS and 1,610 healthy controls were recruited from 8 randomly selected schools, in 4 provinces in Sri Lanka. Data was collected using a previously validated, self administered questionnaire.

\section{Results}

Constipation predominant, diarrhea predominant and mixed type IBS were almost equally distributed (27\%-28\%), while unsubtyped IBS had a lower prevalence (17.8\%). IBS was more common in girls $(59.8 \%$ vs $40.2 \%$ in boys, $P=0.001)$. Bloating, flatulence, burping, headache and limb pain were significantly higher in affected children $(P<0.05)$.

\section{Conclusions}

This study highlights the distribution of IBS subtypes among Sri Lankan children and adolescents and its female preponderance. This study also shows a higher prevalence of other intestinal-related and extraintestinal somatic symptoms among affected children.

(J Neurogastroenterol Motil 2012;18:298-304)

Key Words

Adolescent; Child; Gastrointestinal disorder; Habits; Irritable bowel syndrome

\section{Introduction}

Irritable bowel syndrome (IBS) is a functional gastrointestinal disease (FGID), characterized by abdominal pain and altered bowel habits. Available epidemiological studies have re- ported IBS in $7 \%-14 \%$ of school children. ${ }^{1-3}$ Office-based studies using Rome criteria have found IBS in $21 \%-45 \%$ of children with recurrent abdominal pain. ${ }^{4-7}$ These studies have highlighted the high burden of the disease, both in the community and referral centres. Furthermore, IBS has a significant impact on quality of life of affected children.

Received: December 29, 2011 Revised: February 12, 2012 Accepted: February 23, 2012

(c) This is an Open Access article distributed under the terms of the Creative Commons Attribution Non-Commercial License (http://creativecommons. org/licenses/by-nc/3.0) which permits unrestricted non-commercial use, distribution, and reproduction in any medium, provided the original work is properly cited.

*Correspondence: Shaman Rajindrajith, MBBS, MD, MRCPCH

Department of Pediatrics, Faculty of Medicine, University of Kelaniya, Talagolla Road, Ragama 11010, Sri Lanka

Tel: +0094-11-2958039, Fax: +0094-11-2958337, E-mail: shamanr0@lycos.com

Financial support: This study was supported by the University of Kelaniya, Sri Lanka (Grant No. RP/03/04/03/01/2008).

Conflicts of interest: None. 
Previous adult studies have demonstrated a higher prevalence of IBS in females. ${ }^{9}$ Furthermore, females seek health care for IBS more often than males. ${ }^{10}$ Gender difference in symptomatology and associated features has been overlooked in pediatric studies.

Altered bowel habits (altered bowel frequency and consistency) are among the cardinal features of IBS. Rome III criteria for adults have classified IBS in to constipation predominant IBS (IBS-C), diarrhea predominant IBS (IBS-D), mixed IBS (IBS-M) and unsubtyped IBS (IBS-U), depending on the stool consistency. ${ }^{11}$ However, such a classification has not been specified for IBS in children. ${ }^{12}$ Despite this, a recent study in children has sub-typed IBS to IBS-C, IBS-D, IBS-M and IBS-U using adult criteria. ${ }^{3}$ According to previous studies, the commonest IBS subtype seen in both children and adults was IBS-M. ${ }^{3,13}$

Classification of IBS into relevant subgroup is important since clinical features and managements vary between different subtypes. Many recently developed drugs in adults were developed based on IBS subtype. ${ }^{14-17}$ For example; alosetron was developed to treat IBS-D, ${ }^{14}$ while linaclotide ${ }^{15,16}$ and lubiprostone ${ }^{17}$ were used to treat IBS-C. Therefore, it is fundamental to identify subtypes of IBS in children since most future treatment strategies will target on specific IBS subtype.

The current research was conducted with the objectives of characterizing subtypes of IBS in children and identifying gender differences in the symptomatology. To our knowledge, this is the first pediatric study to analyse bowel habits and symptom characteristics in different IBS subtypes.

\section{Materials and Methods}

This is an island-wide survey, conducted in 8 randomly se- lected schools, in 4 randomly selected provinces (out of 9 provinces) in Sri Lanka. In each school, 2 classes each were selected from academic years (grades) 6-11 (12 classes from each school). All students in selected classes, present during the day of the survey, were included.

Information regarding abdominal pain characteristics, bowel habits and associated symptoms were collected using a self administered questionnaire. The questionnaire was developed based on Rome III diagnostic questionnaire for pediatric functional gastrointestinal disorders ${ }^{18}$ and has previously been pretested for Sri Lankan children and used in an epidemiological survey. ${ }^{3}$ The questionnaire was in native language and easy to understand. School administration and parents were informed before the study and consents were obtained. The questionnaire was distributed in an examination setting to ensure confidentiality and privacy. Children were given unlimited time to fill the questionnaire and verifications were provided by research assistants.

IBS was diagnosed using Rome III criteria for pediatric FGID $^{12}$ and subtyping was done using criteria described by Longstreth et al (Table 1). ${ }^{11}$

Exclusion criteria were (1) functional gastrointestinal disorders other than IBS, (2) chronic disorders needing long term medication other than IBS, (3) disabled children, (4) children with learning difficulties and (5) children who had received drugs that modify bowel habits during previous month. Ethical Review Committee of the Sri Lanka College of pediatricians approved this study protocol.

\section{Statistical Methods}

Data were analysed using EpiInfo (EpiInfo 6, version 6.04 [1996]; Centres of Disease Control and Prevention, Atlanta, Georgia, USA and World Health Organization, Geneva,

Table 1. Diagnostic Criteria for Irritable Bowel Syndrome and Subtypes

\section{Rome III criteria for pediatric IBS ${ }^{12}$}

Abdominal discomfort or pain that occurs at least once per week for more than 2 months associated with at least 2 of the following three features for at least $25 \%$ of the time;

(1) Abdominal pain improved with defecation

(2) Onset associated with change in stool frequency

(3) Onset associated with a change in consistency of stools

Subtypes of IBS $^{11}$

(1) Constipation predominant IBS - hard or lumpy stools $\geq 25 \%$ and loose (mushy) or watery stools $<25 \%$ of bowel movements

(2) Diarrhea predominant IBS - loose (mushy) stools or watery stools $\geq 25 \%$ and hard or lumpy stools $<25 \%$ of bowel movements

(3) Mixed IBS - hard or lumpy $\geq 25 \%$ and loose (mushy) or watery stools $\geq 25 \%$ of bowel movements

(4) Unsubtyped IBS - insufficient abnormality of stool consistency to meet criteria for IBS-C, IBS-D or IBS-M

IBS, irritable bowel syndrome; IBS-C, constipation predominant IBS; IBS-D, diarrhea predominant IBS; IBS-M, mixed IBS. 
Switzerland). A $P<0.05$ was taken as significant. Multiple logistic regression analysis was performed on variables which showed significant associations with IBS during univariate analysis.

\section{Results}

During the survey, 2,180 questionnaires were distributed and all of them were returned. A total of 1,717 children (boys 950 [55.3\%], mean age $13.4 \pm 1.7$ [SD] years) were included in the final analysis. Seventeen incompletely filled questionnaires and 446 children with FGID other than IBS were excluded from the analysis.

Hundred and seven children had IBS (mean age $12.9 \pm 1.8$

Table 2. Subtypes of Irritable Bowel Syndrome According to Sex

\begin{tabular}{lrcc}
\hline \multicolumn{1}{c}{ IBS subtype } & Male & Female & Total \\
\hline IBS-C (n [\%]) & $8(27.6)$ & $21(72.4)^{\mathrm{a}}$ & $29(27.1)$ \\
IBS-D (n [\%]) & $13(43.3)$ & $17(56.7)$ & $30(28.0)$ \\
IBS-M (n [\%]) & $13(44.8)$ & $16(55.2)$ & $29(27.1)$ \\
IBS-U (n [\%]) & $9(47.4)$ & $10(52.6)$ & $19(17.8)$ \\
IBS-Total (n [\%]) & $43(40.2)$ & $64(59.8)^{\mathrm{a}}$ & $107(100.0)$ \\
\hline
\end{tabular}

${ }^{a}$ Female vs male, $P<0.01$ (unpaired $t$ test).

IBS, irritable bowel syndrome; IBS-C, constipation predominant IBS; IBS-D, diarrhea predominant IBS; IBS-M, mixed IBS; IBS-U, unsubtyped IBS.
[SD] years). They were compared with 1,610 children without FGID (mean age $13.5 \pm 1.7$ [SD] years).

\section{Irritable Bowel Syndrome and Sex Distribu- tion}

Table 2 shows the distribution of IBS subtypes according to sex. When mean predicted probabilities of IBS were plotted against the age (Figure), IBS was significantly higher among girls in all age groups $(P<0.01)$. There was a significant neg-

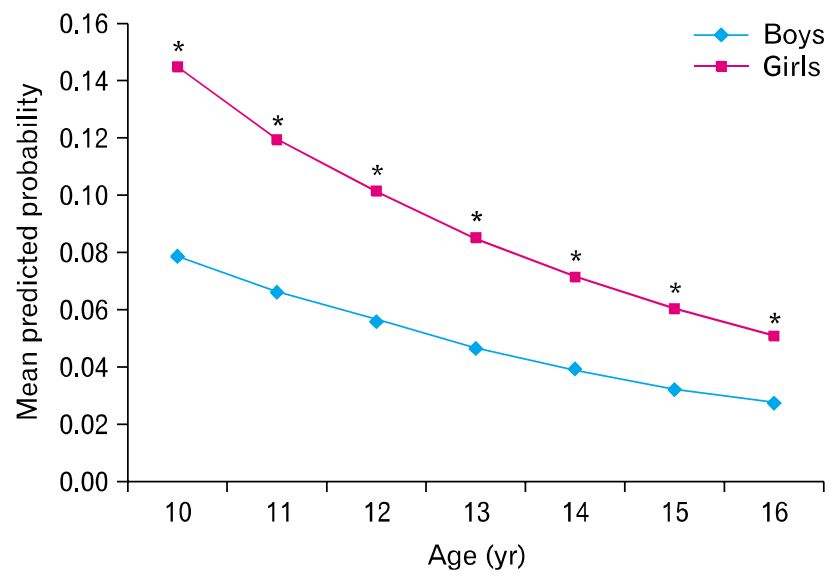

Figure. Mean predicted probabilities of irritable bowel syndrome according to age and sex. ${ }^{*} P<0.01$, Girls vs boys (unpaired $t$ test).

Table 3. Abdominal Pain Characteristics in Children With Irritable Bowel Syndrome

\begin{tabular}{|c|c|c|c|c|c|}
\hline & IBS-C & IBS-D & IBS-M & IBS-U & IBS-Total \\
\hline \multicolumn{6}{|c|}{ Frequency of pain (n [\%]) } \\
\hline Once per wk & $12(41.4)$ & $15(50.0)$ & $10(34.5)$ & $7(36.8)$ & $44(41.1)$ \\
\hline Several times per wk & $14(48.3)$ & $15(50.0)$ & $17(58.6)$ & $12(63.2)$ & $58(54.2)$ \\
\hline Everyday & $3(10.3)$ & $0(0.0)$ & $2(6.9)$ & $0(0.0)$ & $5(4.7)$ \\
\hline \multicolumn{6}{|c|}{ Duration of a pain episodes (n $[\%]$ ) } \\
\hline $1-2 \mathrm{hr}$ & $8(27.6)$ & $9(30.0)$ & $9(31.0)$ & $5(26.3)$ & $31(29.0)$ \\
\hline $3-4 \mathrm{hr}$ & $4(13.8)$ & $0(0.0)$ & $2(6.9)$ & $2(10.5)$ & $8(7.5)$ \\
\hline Most of the day & $4(13.8)$ & $2(6.7)$ & $3(10.3)$ & $0(0.0)$ & $9(8.4)$ \\
\hline \multicolumn{6}{|l|}{ Severity of pain (n [\%]) } \\
\hline Mild & $9(31.0)$ & $4(13.3)$ & $2(6.9)$ & $5(26.3)$ & $20(18.7)$ \\
\hline Moderate & $13(44.8)$ & $25(83.3)$ & $18(62.1)$ & $11(58.0)$ & $67(62.6)$ \\
\hline \multicolumn{6}{|l|}{ Location of pain ( $\mathrm{n}[\%]$ ) } \\
\hline Upper abdomen & $8(27.6)$ & $6(20.0)$ & $8(27.6)$ & $4(21.1)$ & $26(24.3)$ \\
\hline Lower abdomen & $11(37.9)$ & $16(53.3)$ & $11(37.9)$ & $12(63.2)$ & $50(46.7)$ \\
\hline Both & $10(34.5)$ & $8(26.7)$ & $10(34.5)$ & $3(15.7)$ & $31(29.0)$ \\
\hline
\end{tabular}

${ }^{\mathrm{a}} P=0.023$, compared to other 3 types (unpaired $t$ test).

IBS, irritable bowel syndrome; IBS-C, constipation predominant IBS; IBS-D, diarrhea predominant IBS; IBS-M, mixed IBS; IBS-U, unsubtyped IBS. 
ative correlation between probability of developing IBS and age, in both genders (Figure).

Following multiple logistic regression analysis, female sex (adjusted OR, 1.9; 95\% CI, 1.3-2.8, $P=0.002)$ and age (OR, $0.83 ; 95 \% \mathrm{CI}, 0.74-0.93 ; P=0.002)$ remained to be significantly associated with IBS.

\section{Abdominal Pain Characteristics of Irritable Bo- wel Syndrome}

Table 3 shows abdominal pain characteristics in children with IBS. Severe pain was less common in children with IBS-D compared to other 3 subtypes.

\section{Bowel Habits in Children With Irritable Bowel Syn- drome}

Regular bowel habits of children with IBS are shown in Table 4. The bowel habits reported in this table were present during the last 2 months prior to collecting data.

\section{Intestinal-related and Extra-intestinal Symptoms Associated With Irritable Bowel Syndrome}

Intestinal-related symptoms and extra-intestinal symptoms were present in significantly higher proportions in children with all types of IBS (Table 5).
Intestinal-related symptoms (bloating, loss of appetite, nausea, vomiting, flatulence and burping), and extraintestinal symptoms (headache, sleeping difficulties, limb pain and photophobia) were compared between boys and girls with IBS. Only symptom that showed a significant gender difference was burping (boys 24 [56\%] vs girls $16[25 \%], P=0.002)$.

\section{Impact on Education}

Out of 107 children with IBS, 50 (46.7\%) had missed school at least one day during previous 2 months compared to that of 86 (5.3\%) among controls $(P<0.0001$, unpaired $t$ test).

\section{Discussion}

To the best of our knowledge, this is the first pediatric study that has described bowel habits and symptoms according to different subtypes of IBS. In this study, IBS-C, IBS-D and IBS-M were equally distributed, while IBS-U had a lower prevalence. IBS was commoner among females and it negatively correlated with age. Other intestinal-related and extraintestinal symptoms were significantly higher in children with IBS.

IBS is a common abdominal pain predominant FGID among children $^{1-3}$ and adults. ${ }^{19,20}$ A previous pediatric study using Rome III criteria has reported IBS in $7 \%$ of Sri Lankan children. ${ }^{3}$

Table 4. Bowel Habits According to Subtypes of Irritable Bowel Syndrome

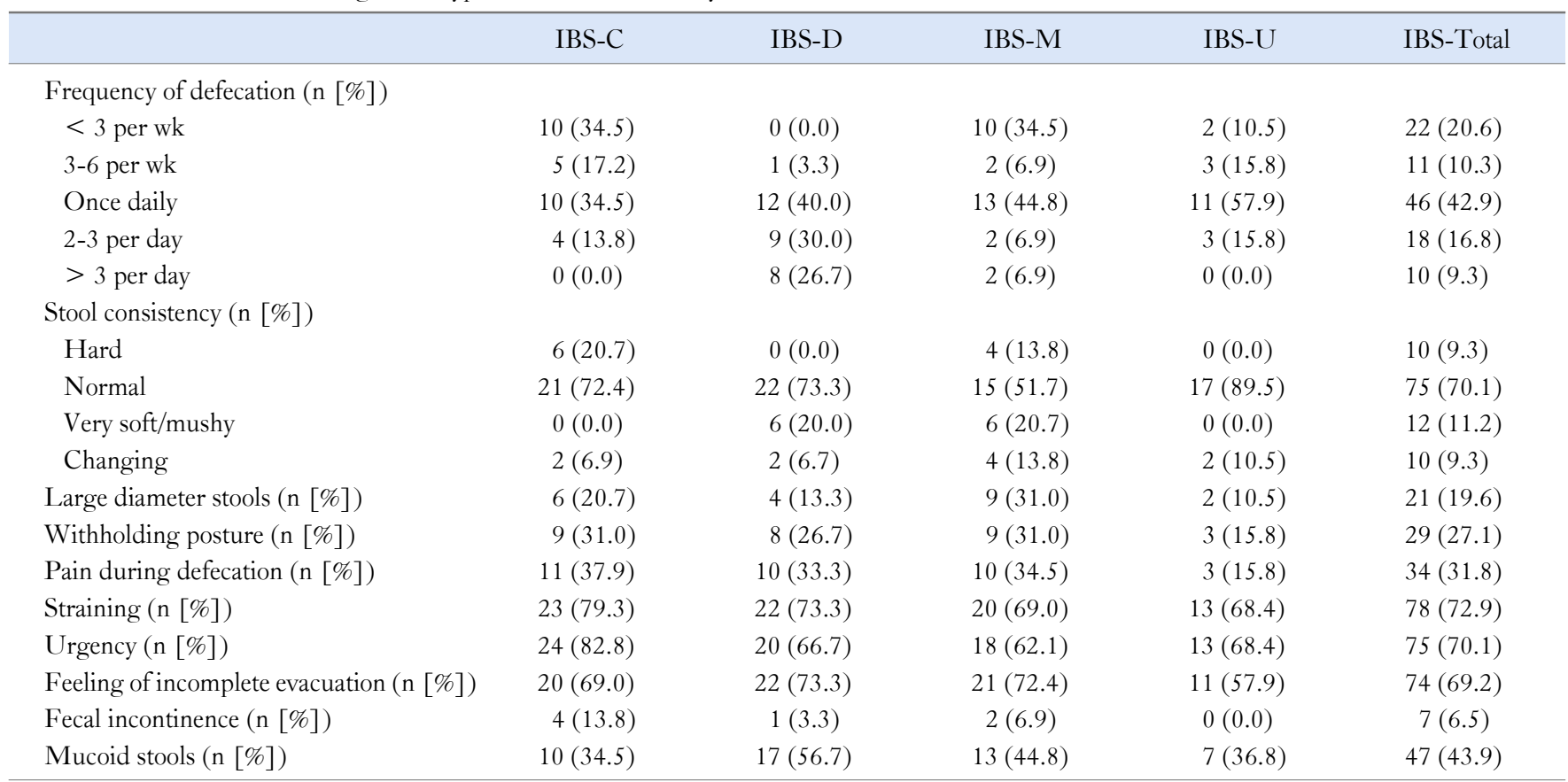

IBS, irritable bowel syndrome; IBS-C, constipation predominant IBS; IBS-D, diarrhea predominant IBS; IBS-M, mixed IBS; IBS-U, unsubtyped IBS. 
Table 5. Intestinal-related and Extraintestinal Symptoms Associated With Irritable Bowel Syndrome

\begin{tabular}{|c|c|c|c|c|c|c|}
\hline & IBS-C & IBS-D & IBS-M & IBS-U & IBS-Total & Controls \\
\hline \multicolumn{7}{|c|}{ Intestinal symptoms (n [\%]) } \\
\hline Bloating & $20(68.9)^{\mathrm{c}}$ & $15(50.0)^{\mathrm{c}}$ & $21(72.4)^{\mathrm{c}}$ & $6(31.6)^{c}$ & $62(57.9)^{\mathrm{c}}$ & $86(5.3)$ \\
\hline Loss of appetite & $7(24.1)^{\mathrm{c}}$ & $7(23.3)^{\mathrm{c}}$ & $11(37.9)^{\mathrm{c}}$ & $6(31.6)^{\mathrm{c}}$ & $31(29.0)^{\mathrm{c}}$ & $75(4.7)$ \\
\hline Nausea & $8(27.6)^{c}$ & $10(33.3)^{c}$ & $8(27.6)^{c}$ & $4(21.1)^{c}$ & $30(28.0)^{c}$ & $49(3.0)$ \\
\hline Vomiting & $6(20.7)^{\mathrm{c}}$ & $3(10.0)^{\mathrm{c}}$ & $7(24.1)^{\mathrm{c}}$ & $1(5.3)$ & $17(15.9)^{\mathrm{c}}$ & $13(0.8)$ \\
\hline Excessive flatus & $19(65.5)^{c}$ & $21(70.0)^{c}$ & $16(55.2)^{\mathrm{c}}$ & $11(57.9)^{\mathrm{c}}$ & $67(62.6)^{c}$ & $254(15.8)$ \\
\hline Burping & $10(34.5)^{\mathrm{a}}$ & $13(43.3)^{\mathrm{b}}$ & $10(34.5)^{\mathrm{a}}$ & $7(36.8)$ & $40(37.3)^{\mathrm{c}}$ & $284(17.6)$ \\
\hline \multicolumn{7}{|c|}{ Extra-intestinal symptoms (n [\%]) } \\
\hline Headache & $17(58.2)^{\mathrm{c}}$ & $13(43.3)^{c}$ & $11(37.9)^{\mathrm{c}}$ & $6(31.6)^{c}$ & $47(43.9)^{\mathrm{c}}$ & $104(6.4)$ \\
\hline Sleeping difficulty & $15(51.7)^{\mathrm{c}}$ & $9(30.0)^{c}$ & $16(55.2)^{\mathrm{c}}$ & $4(21.1)^{c}$ & $44(41.1)^{c}$ & $57(3.5)$ \\
\hline Limb pain & $11(37.9)^{\mathrm{c}}$ & $16(53.3)^{\mathrm{c}}$ & $17(58.6)^{\mathrm{c}}$ & $9(47.4)^{\mathrm{c}}$ & $53(49.5)^{c}$ & $132(8.2)$ \\
\hline Photophobia & $8(27.6)^{c}$ & $7(23.3)^{c}$ & $9(31.0)^{\mathrm{c}}$ & $5(26.3)^{c}$ & $29(27.1)^{c}$ & $30(1.9)$ \\
\hline
\end{tabular}

${ }^{\mathrm{a}} P<0.05,{ }^{\mathrm{b}} P<0.001$ and ${ }^{\mathrm{c}} P<0.0001$ compared to controls (unpaired $t$ test).

IBS, irritable bowel syndrome; IBS-C, constipation predominant IBS; IBS-D, diarrhea predominant IBS; IBS-M, mixed IBS; IBS-U, unsubtyped IBS.

Distribution of IBS subtypes in the present study was similar to that reported previously. ${ }^{3}$ Identification of IBS subtypes is becoming important in clinical practice and research, since pharmacological management of IBS is becoming more specific and most new therapies are developed targeting specific IBS subtypes. Therefore, it is important to use this classification in the future, when evaluating affected children.

In this study, IBS was more common among girls. This is similar to the previous adult studies which have also shown female preponderance. ${ }^{20}$ Differences in activities of male and female gonadal hormone have been suggested as a possible cause for this difference. ${ }^{21}$ However previous pediatric studies failed to show a gender difference in IBS. ${ }^{1-3}$ Small sample size ${ }^{3}$ and younger age of children, ${ }^{2}$ may have contributed to this. We also noted that IBS-C was more prevalent among girls which was similar to an adult study. ${ }^{9}$ Contrary to these findings, another adult study using Rome III criteria have found no gender difference in prevalence of subtypes. $^{22}$

We found a linear reduction in probability of developing IBS as children became older, in both girls and boys. Such correlation has been previously demonstrated in children with defecation disorders such as constipation. ${ }^{23}$

Analysis of symptoms has shown that the majority of affected children had abdominal pain several times a week which lasted for less than one hour. Severity was highest among children with IBS-M (31\%) and it was significantly lower in IBS-D (3.3\%). Previous adult studies have also shown the highest pain severity in IBS-M when using Rome III criteria ${ }^{22}$ and alternating IBS, which is similar to IBS-M type, when using Rome II criteria. ${ }^{24}$
In our study, regular bowel habits of affected children were compatible with IBS subtype. More than one-third of children with IBS-C had bowel motions less than 3 times per week and more than 50\% with IBS-D had opened bowel several times per day. Furthermore, no one in the IBS-C group had very soft or mushy stools and none of the children with IBS-D had hard stools. These observations showed general trend of bowel habits towards constipation or diarrhea were in keeping with the clinical diagnosis. Furthermore, we also found that the supportive symptoms of IBS, such as straining, urgency, feeling of incomplete evacuation and mucoid stools were common in children with all subtypes of IBS. Two previous studies have shown that straining and urgency are common clinical features in children with IBS. ${ }^{1,2}$ However, prevalence of these 2 symptoms, in previous studies, was lower compared to our findings. Contrast to this, adult studies have shown that over $80 \%$ of patients with all subtypes have straining, urgency and sense of incomplete evacuation. ${ }^{24}$ These are troublesome symptoms causing significant distress and should not be overlooked in the clinical evaluation.

Intestinal-related symptoms such as bloating, loss of appetite, nausea, vomiting, flatulence and burping were significantly higher in all IBS subtypes compared to controls. Our results are compatible with previous studies which have shown that bloating is an important problem among both children and adults with IBS. ${ }^{1,25}$ Another study, conducted among adults attending a specialized gastroenterology clinic, has shown that bloating is a predictor of severity of IBS. ${ }^{26}$ Similar to our study, one previous study in patients with IBS-C has shown more bloating in affected individuals. ${ }^{27}$ In the current study, other features of abnormal gastro- 
intestinal gas handling, such as burping and flatulence, were also common among children with IBS. These clinical features have not been studied in details in children with IBS previously. Serra et $\mathrm{al}^{28}$ have demonstrated impaired transit and tolerance of gas in adults with IBS. It is possible that children with IBS have abnormal expulsion of intestinal gas due to altered gastrointestinal motility. Analysis of gender differences showed burping more commonly in boys. Similarly, a previous study in Mexico has reported higher prevalence of burping in males. ${ }^{29}$ Contrary to our finding, previous adult studies have reported bloating more commonly in females with IBS. ${ }^{29,30}$

Extraintestinal symptoms, such as somatic pain and discomfort, are important predictors of disease severity in IBS. ${ }^{26}$ According to adult data, two-thirds of patients with IBS suffer from extraintestinal symptoms. ${ }^{31}$ In the current study, headache, sleeping difficulty, limb pain, and photophobia were significantly commoner among children with all four subtypes of IBS. Similar to our results, Dong et al ${ }^{2}$ also found headache and sleeping difficulty in a higher percentage in affected children. An adult study using Somatic Symptom Checklist has also shown that symptoms such as headache, insomnia and eye pain are common among a community sample of IBS. According to that study, there is a significant association between somatization and IBS. ${ }^{32}$ High prevalence of somatic symptoms in our children with IBS is suggestive of a similar association in pediatric population. Somatization is believed to be more common among females, ${ }^{33,34}$ however we failed to demonstrate any significant symptom predilection to a particular gender. Somatization of our study sample of 10-16 years old is probably different from mature adults and this may have resulted in lack of difference.

This study also demonstrated the impact of IBS on schooling of affected children. Significantly higher percentage of children with IBS has missed school during previous 2 months compared to that of healthy controls. Therefore, it is possible that abdominal pain, altered bowel habits and extraintestinal symptoms may have disturbed daily functions of affected children in our sample, preventing them from attending school.

The main drawback in this questionnaire based survey was the inability to rule out organic disorders. Previous study conducted in Sri Lankan children has reported organic disorders in $11 \%$ of children with recurrent abdominal pain. ${ }^{7}$ We expect a similar percentage of organic disorders in this group of children. Nonetheless, most of the previous epidemiological studies done among adults and children have used a similar methodology. $2,3,20,30,35-37$

In conclusion, this study shows the distribution of IBS sub- types in 10-16 year olds, their symptom characteristics, and bowel habits. IBS-C, IBS-D and IBS-M have almost equal distribution while IBS-U has a relatively lower prevalence. Girls are more commonly affected than boys. Intestinal-related symptoms and extraintestinal symptoms are significantly more common in those with IBS, indicating higher occurrence of somatisation among affected children.

\section{References}

1. Hyams JS, Burke G, Davis PM, Rzepski B, Andrulonis PA. Abdominal pain and irritable bowel syndrome in adolescents: a community-based study. J Pediatr 1996;129:220-226.

2. Dong L, Dingguo L, Xiaoxing X, Hanming L. An epidemiologic study of irritable bowel syndrome in adolescents and children in China: a school-based study. Pediatrics 2005;116:e393-e396.

3. Devanarayana NM, Adhikari C, Pannala W, Rajindrajith S. Prevalence of functional gastrointestinal diseases in a cohort of Sri Lankan adolescents: comparison between Rome II and Rome III criteria. J Trop Pediatr 2011;57:34-39.

4. Caplan A, Walker L, Rasquin A. Validation of the pediatric Rome II criteria for functional gastrointestinal disorders using the questionnaire on pediatric gastrointestinal symptoms. J Pediatr Gastroenterol Nutr 2005;41:305-316.

5. Walker LS, Lipani TA, Greene JW, et al. Recurrent abdominal pain: symptom subtypes based on the Rome II Criteria for pediatric functional gastrointestinal disorders. J Pediatr Gastroenterol Nutr 2004;38:187-191.

6. Helgeland H, Flagstad G, Grøtta J, Vandvik PO, Kristensen H, Markestad T. Diagnosing pediatric functional abdominal pain in children (4-15 years old) according to Rome III Criteria: results from a Norwegian prospective study. J Pediatr Gastroenterol Nutr 2009;49:309-315.

7. Devanarayana NM, de Silva DG, de Silva HJ. Aetiology of recurrent abdominal pain in a cohort of Sri Lankan children. J Paediatr Child Health 2008;44:195-200.

8. Vani JW, Lane MM, Burwinkle TM, et al. Health-related quality of life in pediatric patients with irritable bowel syndrome: a comparative analysis. J Dev Behav Pediatr 2006;27:451-458.

9. Rey E, Talley NJ. Irritable bowel syndrome: novel views on the epidemiology and potential risk factors. Dig Liver Dis 2009;41:772780 .

10. Talley NJ, Boyce PM, Jones M. Predictors of health care seeking for irritable bowel syndrome: a population based study. Gut 1997;41: 394-398.

11. Longstreth GF, Thompson WG, Chey WD, Houghton LA, Mearin F, Spiller RC. Functional bowel disorders. Gastroenterology 2006;130:1480-1491.

12. Rasquin A, Di Lorenzo C, Forbes D, et al. Childhood functional gastrointestinal disorders: child/adolescent. Gastroenterology 2006; 130:1527-1537.

13. Videlock EJ, Chang L. Irritable bowel syndrome: current approach to symptoms, evaluation, and treatment. Gastroenterol Clin North 
Am 2007;36:665-685.

14. Camilleri M, Chey WY, Mayer EA, et al. A randomized controlled clinical trial of the serotonin type 3 receptor antagonist alosetron in women with diarrhea-predominant irritable bowel syndrome. Arch Intern Med 2001;161:1733-1740.

15. Johnston JM, Kurtz CB, Macdougall JE, et al. Linaclotide improves abdominal pain and bowel habits in a phase IIb study of patients with irritable bowel syndrome with constipation. Gastroenterology 2010; 139:1877-1886.

16. Johnston JM, MacDougall JE, Lavins B, et al. Linaclotide significantly improved abdominal pain, constipation and global assessments in adults with irritable bowel syndrome with constipation: results from a large twelve week, randomised, double-blind, placebo-controlled study. Am J Gastroenterol 2008;103:S460-S461.

17. Drossman DA, Chey WD, Johanson JF, et al. Clinical trial: lubiprostone in patients with constipation-associated irritable bowel syndrome- results of two randomized, placebo-controlled studies. Aliment Pharmacol Ther 2009;29:329-341.

18. Walker LS, Caplan A, Rasquin A. Rome III diagnostic questionnaire for the pediatric functional GI disorders. In: Drossman DA, Corazziari E, Delvaux N, et al, eds. Rome III: the functional gastrointestinal disorders. McLean, VA: Degnon Associates 2006: 961-990.

19. Gwee KA. Irritable bowel syndrome in developing countries - a disorder of civilization or colonization? Neurogastroenterol Motil 2005; 17:317-324.

20. Hungin AP, Chang L, Locke GR, Dennis EH, Barghout V. Irritable bowel syndrome in the United States: prevalence, symptom patterns and impact. Aliment Pharmacol Ther 2005;21:1365-1375.

21. Heitkemper MM, Jarrett ME. Update on irritable bowel syndrome and gender differences. Nutr Clin Pract 2008;23:275-283.

22. Ersryd A, Posserud I, Abrahamsson H, Simrén M. Subtyping the irritable bowel syndrome by predominant bowel habit: Rome II versus Rome III. Aliment Pharmocol Ther 2007;26:953-961.

23. Rajindrajith S, Devanaryana NM, Adhikari C, Pannala W, Benninga MA. Constipation in children: an epidemiological study in Sri Lanka using Rome III criteria. Arch Dis Child 2012;97:43-45.

24. Tillisch K, Labus JS, Naliboff BD, et al. Characterization of the alternating bowel habit subtype in patients with irritable bowel syndrome. Am J Gastroenterol 2005;100:896-904.

25. Lembo T, Naliboff B, Munakata J, et al. Symptoms and visceral perception in patients with pain-predominant irritable bowel syndrome.
Am J Gastroenterol 1999;94:1320-1326.

26. Spiegel B, Strickland A, Naliboff BD, Mayer EA, Chang L. Predictors of patient-assessed illness severity in irritable bowel syndrome. Am J Gastroenterol 2008;103:2536-2543.

27. Agrawal A, Houghton LA, Reilly B, Morris J, Whorwell PJ. Bloating and distension in irritable bowel syndrome: the role of gastrointestinal transit. Am J Gastroenterol 2009;104:1998-2004.

28. Serra J, Azpiroz F, Malagelada JR. Impaired transit and tolerance of intestinal gas in the irritable bowel syndrome. Gut 2001;48:14-19.

29. Schmulson M, Adeyemo M, Gutiérrez-Reyes G, et al. Differences in gastrointestinal symptoms according to gender in Rome II positive IBS and dyspepsia in a Latin American population. Am J Gastroenterol 2010;105:925-932.

30. Ringel Y, Williams RE, Kalilani I, Cook SF. Prevalence, characteristics, and impact of bloating symptoms in patients with irritable bowel syndrome. Clin Gastroenterol Hepatol 2009;7:68-72.

31. Whitehead WE, Palsson O, Jones KR. Systematic review of the comorbidity of irritable bowel syndrome with other disorders: what are the causes and implications? Gastroenterology 2002;122:1140-1156.

32. Choung RS, Locke GR 3rd, Zinsmeister AR, Schleck CD, Talley NJ. Psychosocial distress and somatic symptoms in community subjects with irritable bowel syndrome: a psychological component is the rule. Am J Gastroenterol 2009;104:1772-1779.

33. Rief W, Hessel A, Braehler E. Somatization symptoms and hypochondriacal features in the general population. Psychosom Med 2001; 63:595-602.

34. Ladwig KH, Marten-Mittag B, Erazo N, Gundel H. Identifying somatization disorder in a population-based health examination survey: psychosocial burden and gender differences. Psychosomatics 2001;42:511-518.

35. Hillilä MT, Färkkilä MA. Prevalence of irritable bowel syndrome according to different diagnostic criteria in a non-selected adult population. Aliment Pharmacol Ther 2004;20:339-345.

36. Hungin AP, Whorwell PJ, Tack J, Mearin F. The prevalence, patterns and impact of irritable bowel syndrome: an international survey of 40,000 subjects. Aliment Pharmacol Ther 2003;17:643-650.

37. Sohrabi S, Nouraie M, Khademi H, Baghizadeh S, Nasseri-Moghaddam S, Malekzadeh R. Epidemiology of uninvestigated gastrointestinal symptoms in adolescents: a population-based study applying the Rome III questionnaire. J Pediatr Gastroenterol Nutr 2010; 51:41-45. 